\title{
Proposition d'un modèle conceptuel du traumatisme vicariant appliqué à la gestion des ressources humaines : le cas des conseillers en accompagnement vers l'emploi
}

\author{
Julien CUSIN \\ IAE Bordeaux, Université Bordeaux \\ IRGO \\ Catherine FABRE \\ IAE Bordeaux, Université Bordeaux \\ IRGO
}

\section{INTRODUCTION}

S'il est connu que la violence des échanges peut parfois conduire au mal-être psychologique des individus au travail (Ben-Zur et Yagil, 2005 ; Grandey et al., 2004), l'idée selon laquelle la bienveillance peut aussi conduire à la souffrance au travail est moins évidente. Cet article propose de décrire et de comprendre ces situations particulières, où un individu est fragilisé psychologiquement du fait de l'empathie dont il fait preuve dans le cadre de son travail auprès de personnes en souffrance. A ce jour, la littérature en gestion a déjà mis en lumière un phénomène de transmission positive comme l'apprentissage vicariant (Bresman, 2013; Tuschke et al., 2014) ${ }^{1}$. En revanche, elle ne s'est pas encore intéressée au phénomène de contagion négative qu'est le traumatisme vicariant, qui désigne, dans les revues médicales, le fait d'être durablement marqué psychologiquement, du fait de l'expérience douloureuse vécue par d'autres auprès de qui l'on travaille (McCann et Pearlman, 1990 ; Pearlman et Saakvitne, 1995). Ce manque paraît surprenant, dans la mesure où les entreprises sont de plus en plus sensibilisées aux risques psychosociaux du travail et où leur responsabilité dans la santé mentale de leurs salariés est questionnée par les pouvoirs publics (Dejours, 2007 ; Bué, 2008). Pour étudier le concept de traumatisme vicariant, nous avons choisi de nous focaliser sur un type particulier de métier, qui nous semble caractéristique à la fois du point de vue du

\footnotetext{
${ }^{1}$ L'apprentissage vicariant désigne le fait d'acquérir des compétences, de manière plus ou moins consciente, par l'observation ou par l'imitation.
} 
traumatisme ressenti par les clients, et du haut niveau d'empathie requis : le secteur de l'accompagnement à l'insertion professionnelle. En effet, malgré la banalisation des ruptures professionnelles et le constat de la fin des carrières linéaires (Aquilanti et Leroux, 1999; Cadin et al., 2003 ; Dany, 1997 ; London, 1998 ; Pralong, 2011, Roques et Roger, 2004, Sadeh et Karniol, 2012), le fait de perdre son emploi ou de faire faillite (McKee-Ryan et al., 2009 ; Ucbasaran et al., 2013) reste source de grandes souffrances psychologiques. Ces expériences peuvent même provoquer des trauma émotionnels pour les personnes concernées (Gowan et Gatewood, 1997 ; Latack et al., 1995), notamment lorsque le chômage s'éternise (McKee-Ryan et al., 2005 ; Soper et Von Bergen, 2001 ; Spera et al., 1994). De ce fait, le travail psychologique est au cœur de l'accompagnement professionnel, et la première phase d'une démarche d'accompagnement consiste, le plus souvent, à regagner l'équilibre sur le plan psychologique, à travers une relation étroite avec les candidats (Aquilanti et Leroux, 1989 ; Kirk, 1994).

La question se pose alors de savoir comment les conseillers professionnels perçoivent le traumatisme des bénéficiaires, qui est central dans la relation accompagnant/accompagné. En particulier, leur bien-être psychologique est-il affecté par la souffrance accueillie et, dans le cas contraire, pourquoi en sont-ils préservés? Le premier objectif de cet article sera donc d'explorer l'existence potentielle d'un type particulier de souffrance au travail, qui serait dû au fait d'accompagner des personnes ayant subi un traumatisme d'ordre professionnel. Le deuxième objectif de notre recherche consistera à identifier les particularités de l'accompagnement professionnel sur la question de la souffrance au travail, dans la mesure où dans la littérature sur le burnout comme dans celle plus large de la souffrance au travail, l'étude des métiers de l'insertion professionnelle n'est pas centrale. Enfin, emprunter un concept à la littérature médicale pour l'adapter aux enjeux de la fonction RH constituera la troisième contribution attendue de cette recherche. 
Ainsi, en nous appuyant sur le cadre théorique du traumatisme vicariant, nous proposons de construire un modèle conceptuel permettant de comprendre dans quelle mesure le conseiller en accompagnement professionnel souffre de sa relation avec des personnes traumatisées par un choc professionnel et peut y faire face.

Dans cette perspective, il apparaît nécessaire d'adopter une approche qualitative, dans la lignée d'auteurs tels que Clot (2010) ou Dejours (1998, 2010). En effet, celle-ci permet de prendre en considération et de mettre en perspective un grand nombre d'éléments de contexte. On cherchera notamment à vérifier l'existence du traumatisme vicariant, à identifier les différentes formes qu'il est susceptible de prendre, les facteurs permettant de comprendre son apparition ainsi que les leviers individuels et organisationnels permettant de s'en prémunir.

\section{REVUE DE LA LITTERATURE}

Afin de bien cerner l'objet de cette étude, nous passerons en revue les travaux portant sur la souffrance au travail liée à l'empathie dans le domaine de la recherche en gestion des ressources humaines, dans un premier temps, puis nous intéresserons au concept de traumatisme vicariant importé de la littérature médicale ensuite.

\subsection{LA SOUFFRANCE AU TRAVAIL LIEE A L'EMPATHIE EN GRH}

La littérature sur la souffrance psychologique au travail s'est longtemps confondue avec l'étude du stress au travail. En prenant en compte à la fois les contraintes extérieures et les prédispositions individuelles à certains risques psycho-sociaux (Lhuilier, 2010), certains travaux fondateurs se sont attelés à définir des conditions favorisant la survenance du stress au travail. Selon le modèle « exigence-contrôle » de Karasek (1979), le stress résulte ainsi de la coexistence d'exigences du travail fortes et d'une faible latitude décisionnelle du salarié. A titre d'illustration, les derniers rapports sur la souffrance au travail en France (Debout, 1999 ; 
Dejours, 2007 ; Bué, 2008) montrent que la violence des usagers ne suffit pas à expliquer la survenance du mal-être au travail, tandis que son couplage avec une organisation du travail de plus en plus contraignante, associant demande de forte productivité et manque de moyens, contribue à l'augmentation des souffrances psychologiques au travail. Bien qu'ils soient très instructifs pour la compréhension de la survenue du stress au travail, ces travaux n'abordent pas directement la question de la souffrance due à un excès d'empathie vis-à-vis de personnes elles-mêmes en grande détresse.

De ce point de vue, il parait plus fécond de se tourner vers la littérature sur le burnout. En effet, d'après Shaufeli et Greenglas (2001, p.501), «burnout may be defined as a state of physical, emotional and mental exhaustion that results from long-term involvement in work situations that are emotionally demanding. ". Ainsi, caractérisés notamment par le fait qu'ils imposent un certain don de soi, très coûteux en énergie (Day et Chambers, 1991; Kahn, 1993), les secteurs d'activité tels que l'éducation, la santé et les services sociaux ont été les premiers à focaliser l'attention des recherches sur le «burnout» (Maslach, 1982). Ce dernier a donc été d'abord appréhendé comme une «maladie du don », réservée aux professions centrées sur la relation d'aide (Peterson et al., 2008, Ide, 2015). Certaines études sur les personnels soignants ont attesté, par exemple, d'une corrélation entre la fréquence des contacts avec les patients ou la lourdeur de leurs problèmes et le burnout (Lewiston et al., 1981 ; Meadow, 1981). Cependant, il est aujourd'hui établi que le burnout est un phénomène bien plus large, qui peut intervenir du fait d'une multitude de facteurs non liés à l'empathie (Cordes et Dogherty, 1993). Il est le fruit d'une combinaison d'éléments à la fois personnels, organisationnels et liés au travail en lui-même : surcharge de travail, objectifs irréalistes/mal définis, interruptions fréquentes, échanges agressifs ou violents avec les clients ou collègues, obligation d'afficher un sentiment en contradiction avec son sentiment réel, manque d'autonomie, mauvaises 
relations de travail, insécurité socioéconomique (Alis, 2010; Clot, 1995, 2008 ; Dejours, 2007, 2010), etc.

Au final, le burnout est un phénomène large, qui peut apparaître dans une multitude de secteurs d'activités et qui se traduit par diverses manifestations (Demerouti et al., 2001 ; Pezet Langevin, 2002). Ce terme est donc trop diffus et générique pour bien représenter le phénomène que nous étudions ici. Afin de pouvoir focaliser notre analyse sur la transmission de la souffrance d'un individu traumatisé par un choc professionnel à un autre individu qui l'accompagne, il nous paraît donc nécessaire d'importer un concept issu de la médecine : celui de traumatisme vicariant.

\subsection{LE TRAUMATISME VICARIANT EN MEDECINE}

La littérature médicale a établi qu'en étant exposés - de façon prolongée et/ou répétée - à des récits explicites de souffrance (ex. : abus sexuels, violence familiale, etc.), les thérapeutes, cliniciens ou autres travailleurs sociaux peuvent être affectés de façon indirecte (Beck, 2011 ; Figley, 1995 ; Jenkins et al., 2011 ; Shiri et al., 2010 ; Sinclair et Hamill, 2007 ; Trippany et al., 2004 ; Way et al., 2004). Ils sont alors victimes - à un degré moindre - des mêmes maux que les individus directement affectés par l'expérience douloureuse (Beck, 2011 ; Choi, 2011 ; Sinclair et Hamill, 2007). Ce phénomène a reçu une grande attention à partir des années 90 et a été qualifié de «traumatisme vicariant» (McCann et Pearlman, 1990). Pearlman et Saakvitne (1995: p. 280) le définissent de la façon suivante : « [It is a cumulative process] through which the therapist's inner experience is negatively transformed through empathic engagement with the clients' trauma material. ». Pour les auteurs, le traumatisme vicariant est lié à l'engagement empathique, inhérent à la relation qu'un professionnel peut entretenir avec des personnes victimes de traumatismes (Choi, 2011 ; McCann et Pearlman, 
1990 ; Pearlman et Saakvitne, 1995 ; Sinclair et Hamill, 2007 ; Trippany et al., 2004 ; Way et al., 2004).

Par ailleurs, la littérature fait apparaître de multiples facteurs de risque (Choi, 2011 ; Devilly et al., 2009 ; Eriksen et Ditrich, 2015 ; Sabin-Farrel et Turpin, 2003 ; Sinclair et Hamill, 2007 ; Vrklevski et Franklin, 2008 ; Way et al., 2004). Ils sont liés notamment à l'individu lui-même (âge, genre, niveau de formation, statut socio-économique, vision du monde, spiritualité, histoire personnelle, soutien social, contexte de vie actuel, mécanismes personnels de coping, nombre d'années passées à travailler face à des publics en situation de traumatisme, etc.) ou aux facteurs organisationnels (contexte dans lequel le thérapeute exerce (heures de travail, ne pas avoir de limites de temps, etc.), soutien des pairs, supervision, accès à l'information et aux ressources, niveau d'exposition au traumatisme, charge de travail, etc.). Ces facteurs de risque peuvent tout aussi bien être envisagés comme un manque de barrières de protection pour l'individu, signalant en creux qu'il existe des moyens de faire face à un matériel traumatique.

Au plan théorique, le traumatisme vicariant est appréhendé à travers la théorie constructiviste du développement de soi (Elwood et al., 2011 ; McCann et Pearlman, 1990 ; Pearlman et Saakvitne, 1995). Selon cette perspective, les changements liés au traumatisme secondaire ${ }^{2}$ sont intrinsèques à l'individu. Ils comprennent notamment : une perturbation de l'identité de soi et professionnelle; une modification du cadre de référence ; une diminution du sentiment de sécurité, de pouvoir, de contrôle et d'indépendance pour soi-même et ses proches ; des problèmes d'estime de soi et de sentiment d'auto-efficacité, etc. (Elwood et al., 2011 ; Jenkins et al., 2011 ; Pearlman et Saakvitne, 1995 ; Sinclair et Hamill, 2007 ; Trippany et al., 2004 ; Way et al., 2004). Ainsi, les changements cognitifs à long terme constituent la caractéristique

\footnotetext{
${ }^{2}$ Le traumatisme primaire étant celui de l'individu ayant été directement affecté par l'expérience douloureuse.
} 
déterminante du traumatisme vicariant (Elwood et al., 2011 ; Pearlman et Saakvitne, 1995 ; Sabin-Farrell et Turpin, 2003 ; Shiri et al., 2010 ; Sinclair et Hamill, 2007 ; Way et al., 2004). Dans la littérature médicale, le concept de traumatisme vicariant est systématiquement associé à celui de fatigue de compassion, appelé aussi stress traumatique secondaire (Figley, 1995). Celle-ci correspond aux symptômes et aux réponses émotionnelles résultant d'un travail auprès de victimes de traumatisme (Sabin-Farrell et Turpin, 2003). À ce titre, Gentry et al. (2002: p. 126) ont résumé les différents symptômes de stress traumatique secondaire identifiés dans la littérature: augmentation de l'excitation négative, pensées/images intrusives, insomnie, difficulté à séparer le travail de la vie personnelle, tolérance moins grande à la frustration, augmentation des accès de colère ou de rage, peur de travailler avec certaines personnes, dépression, comportements apaisants inefficaces ou autodestructeurs, hyper-vigilance, diminution du sens et du plaisir lié à la carrière, perte d'espoir, etc. Toutefois, la frontière entre les changements cognitifs et la détresse symptomatique reste assez floue dans la littérature, au point que ces terminologies sont parfois utilisées de façon interchangeable (Elwood et al., 2011 ; Sabin-Farrell et Turpin, 2003 ; Sinclair et Hamill, 2007 ; Vrklevski et Franklin, 2008).

Dans une perspective RH, visant à prévenir les RPS, il paraît important de compléter l'analyse de la détresse cognitive par l'identification de symptômes observables. Dans la mesure où il s'agit ici de transposer un concept existant dans le domaine médical à l'univers des sciences de gestion, où il est ignoré à ce jour, nous choisissons d'inclure dans l'étude du traumatisme vicariant l'ensemble de ces dimensions. 


\section{METHODOLOGIE}

\subsection{RECUEIL DES DONNEES}

Pour répondre à notre question de recherche, nous avons opté pour une étude qualitative de cas multiples, et ciblé des conseillers intervenant au sein de structures qui accompagnent vers l'emploi des personnes en situation de rupture (licenciement, dépôt de bilan, chômage longue durée, etc.). En effet, au regard de la littérature (McKee-Ryan et al., 2009 ; Ucbasaran et al., 2013), on peut supposer que certains bénéficiaires de ces dispositifs portent un lourd matériel traumatique. En pratique, notre logique d'échantillonnage est analogue à celle de Balzani et

al. (2008: p. 7): «L'échantillon n'a pas vocation à une quelconque représentativité statistique de l'ensemble des structures spécialisées dans l'accompagnement vers ou dans l'emploi. Il est constitué de structures variées afin d'embrasser le champ de l'accompagnement dans sa multiplicité et sa complexité. ». Or, les structures de l'accompagnement professionnel relèvent tant du secteur public (Pôle Emploi, Maisons de l'emploi, Missions locales, Cap Emploi, etc.) que du secteur privé (cabinets de conseil RH, agences spécialisées dans le placement et/ou le coaching, ETT/ETTI, associations, etc.). En outre, les dispositifs diffèrent quant à la durée de l'accompagnement (limité dans le temps $v s$. illimité), aux caractéristiques des publics (faible vs. forte employabilité), aux objectifs prescrits de l'accompagnement (taux élevé de placement $v s$. aucun objectif de placement), au volontariat du public (accompagnement obligatoire $v s$. libre adhésion), au pouvoir de sanction du conseiller (aucun pouvoir de sanction vs. pouvoir de sanction formel) ou à la proximité avec les offreurs d'emploi (aucun contact avec les employeurs $v s$. capital social important). Nous avons donc cherché à observer un maximum de variabilité dans les contextes d'intervention des personnes interrogées.

En définitive, nous avons interrogé 23 conseillers issus de 13 structures distinctes (cf. annexe $\mathrm{N}^{\circ}$ 1), principalement au sein de Bordeaux et sa métropole, la collecte de données ayant été 
arrêtée à partir du moment où il y a eu saturation du terrain (Yin, 1990). Afin de nous assurer de la pertinence du profil des répondants au regard de notre objet de recherche, nous avons posé une question filtre en début d'entretien, vérifiant la présence d'une souffrance chez les publics accompagnés.

Les structures d'accompagnement ont certes constitué notre point d'entrée sur le terrain, mais l'unité d'analyse retenue dans cette recherche est bien le conseiller en accompagnement vers l'emploi. Il ne s'agit donc pas ici de comparer les structures entre elles, mais bien le ressenti des différents répondants face à des publics en souffrance, ainsi que les conséquences de leur rôle d'accompagnant de personnes traumatisées sur leur vie professionnelle et personnelle. Précisons ici que les 23 entretiens, d'une durée moyenne de $1 \mathrm{H} 23$, ont tous été enregistrés puis retranscrits, ce qui correspond finalement à un corpus de données brutes de 311 pages.

\subsection{ANALYSE DES DONNEES}

Pour traiter ces données, nous avons eu recours à la méthodologie dite 'à la Gioia' (Gioia et al., 2013), de plus en plus mobilisée en sciences de gestion (Monin et Rüling, 2013). À ce jour, cette approche est encore assez peu utilisée dans le champ des RH, même s'il existe des exceptions notables telles que l'article de Corley (2004), par exemple. Comme le résume Reay (2014 : p. 99) : «In this approach, researchers show their analytic work through a boxes and arrows figure that identifies data extracts grouped together according to firstorder codes that are then grouped into second-order themes at a higher level of abstraction ». Le tableau $\mathrm{N}^{\circ} 1$ ci-dessous montre ainsi comment nous sommes passés de 82 items de $1^{\text {er }}$ rang à 13 thèmes de $2^{\text {nd }}$ rang, puis à 4 dimensions agrégées de $2^{\text {nd }}$ rang. Le codage de premier rang est inductif, dans la mesure où son but est de restituer, de la manière la plus fidèle possible, le discours des répondants. En d'autres termes, lors de cette première étape, nous sommes partis des retranscriptions des entretiens, en occultant la littérature. À chaque fois que nous 
repérions une nouvelle idée, nous générions un nouvel item, celui-ci étant in fine conservé s’il était présent chez, au moins, trois de nos répondants (cf. infra). Dans une deuxième étape, le codage de second rang consiste à agréger ces items, à travers deux niveaux d'abstraction successifs, en opérant cette fois-ci des allers-retours entre la théorie et le terrain, dans une démarche abductive. A titre d'illustration, des concepts clefs de notre modèle, tels que le matériel traumatique, le désengagement professionnel ou encore la contagion émotionnelle sont issus d'un retour à la littérature et ont été rattachés, dans un deuxième temps, aux phénomènes observés empiriquement. A l'inverse, la notion de périmètre, par exemple, est totalement émergente. 
Tableau N$^{\circ}$ : Grille Gioia et al. (2013) du traumatisme vicariant

\begin{tabular}{|c|c|c|}
\hline Codage ${ }^{\text {er }}$ rang (inductif) & Codage $2^{\text {nd }}$ rang (abductif) & $\begin{array}{c}\text { Codage de second rang } \\
\text { agrégé }\end{array}$ \\
\hline $\begin{array}{l}\text { - Les bénéficiaires sont en situation de rupture (professionnelle, financière, familiale, etc.) - } 17 \\
\text { - Les bénéficiaires ont traversé des épisodes violents dans leur parcours de vie - } 10 \\
\text { - Les bénéficiaires sont confrontés à des freins périphériques dans leur recherche d'emploi - } 13 \\
\end{array}$ & $\begin{array}{l}\text { [1.1.] Faits marquant le vécu et la } \\
\text { situation actuelle des } \\
\text { bénéficiaires }\end{array}$ & \multirow{3}{*}{$\begin{array}{l}\text { [1.] Matériel traumatique du } \\
\text { bénéficiaire }\end{array}$} \\
\hline $\begin{array}{l}\text { - L'intensité de la souffrance est variable selon les bénéficiaires - } 16 \\
\text { - Le bénéficiaire verbalise ses difficultés (avec plus ou moins de facilite)/"vide son sac"/se décharge - } 14 \\
\text { - Le bénéficiaire est agressif/en colère/sur la défensive/a un discours très négatif - } 9 \\
\text { - Le bénéficiaire pleure durant l'entretien, voire est en dépression - } 14 \\
\text { - Le bénéficiaire menace de se suicider - } 3 \\
\text { - La souffrance s'observe à travers un laisser-aller général, les traits du visage, la gestuelle, le débit de la voix, etc. - } 10\end{array}$ & $\begin{array}{l}\text { [1.2.] Expression verbale ou non } \\
\text { verbale de sa souffrance par le } \\
\text { bénéficiaire }\end{array}$ & \\
\hline $\begin{array}{l}\text { - Le bénéficiaire ressasse les mêmes sujets / son projet piétine ou manque de cohérence - } 7 \\
\text { - Le bénéficiaire est dans l'évitement (ex. : RDV annulés, refus de participer à certains ateliers, etc.) - } 6 \\
\text { - Le bénéficiaire manque de confiance en lui/se dévalorise - } 5\end{array}$ & $\begin{array}{l}\text { [1.3.] Influence de la souffrance } \\
\text { du bénéficiaire sur ses attitudes et } \\
\text { comportements }\end{array}$ & \\
\hline $\begin{array}{l}\text { - Le conseiller repère des limites de compétences dans son accompagnement - } 11 \\
\text { - Le conseiller refuse de jouer le rôle de psychologue - } 7 \\
\text { - Le conseiller est dans l'opérationnalité (ex. : outils, conseils pratiques, démarches administratives, axes de progrès, etc.) - } 7 \\
\text { - Le conseiller prend en compte les problématiques sociales/les freins périphériques, mais ne les traite pas lui-même - } 5 \\
\text { - Le conseiller recentre les échanges avec le bénéficiaire sur le projet professionnel/sur des aspects techniques - } 5\end{array}$ & $\begin{array}{l}\text { [2.1.] Périmètre professionnel de } \\
\text { l'accompagnement }\end{array}$ & \multirow{3}{*}{ [2.] Posture du conseiller } \\
\hline $\begin{array}{l}\text { - Le conseiller fixe les règles de la relation dès le départ - } 8 \\
\text { - Le conseiller est le plus disponible possible pour le bénéficiaire - } 7 \\
\text { - Le conseiller s'adapte aux besoins du bénéficiaire - } 12 \\
\text { - Le conseiller « tient conseil » et fait en sorte que le bénéficiaire soit autonome / prenne lui-même les rênes de son projet - } 12 \\
\text { - Le conseiller crée un lien étroit avec le bénéficiaire et établit une relation de confiance - } 12 \\
\text { - Le conseiller garde une distance avec le bénéficiaire (vouvoiement, pas de relation privée, pas d'intrusion dans la vie privée) - } 9 \\
\text { - Le conseiller écoute le bénéficiaire et accueille sa souffrance (empathie) - } 10 \\
\text { - Le conseiller aide les bénéficiaires, en écho à ses valeurs/croyances - } 9 \\
\text { - Le conseiller fait profiter de sa propre expérience face aux difficultés (il rassure, libère la parole, conseille, etc.) - } 10\end{array}$ & $\begin{array}{l}\text { [2.2.] Périmètre relationnel de } \\
\text { 1'accompagnement }\end{array}$ & \\
\hline $\begin{array}{l}\text { - Le conseiller a plus d'affinités/d'implication avec certains bénéficiaires (notamment les plus motivés) - } 10 \\
\text { - Le conseiller prend du recul / de la distance sur la situation du bénéficiaire / relativise / se détache - } 10 \\
\text { - Le conseiller vit des situations de projection ou d'effet miroir avec le bénéficiaire - } 19 \\
\text { - Le conseiller ne veut pas apparaître comme un sauveur - } 9 \\
\text { - Le conseiller doit accepter le fait de ne pas toujours réussir à être utile pour tous les bénéficiaires - } 10 \\
\text { - Le conseiller doit trouver son propre dosage empathie/distance - 11 } \\
\text { - Le conseiller doit se distancer du point de vue négatif du bénéficiaire pour l'aider à construire un projet positif - } 12\end{array}$ & $\begin{array}{l}\text { [2.3.] Périmètre affectif de } \\
\text { l'accompagnement }\end{array}$ & \\
\hline $\begin{array}{l}\text { - Un rythme d'accompagnement non contraignant (durée des entretiens/suivi) permet de mieux vivre l'accompagnement - } 11 \\
\text { - Le fait d'être dans un dispositif bénéficiant de beaucoup de moyens permet de mieux vivre l'accompagnement - } 6 \\
\text { - Le conseiller peut avoir des objectifs / des contraintes de rentabilité influençant la manière dont il vit l'accompagnement - } 8 \\
\text { - Une forte fréquence d'exposition (ex. : taille du portefeuille) rend l'accompagnement plus difficile à supporter - } 13 \\
\text { - La polyvalence et le cumul d'activités permettent de mieux vivre l'accompagnement - } 8 \\
\text { - Les perspectives d'évolution en dehors du dispositif permettent de mieux vivre l'accompagnement - } 7\end{array}$ & [3.1.] Flexibilité du dispositif & [3.] Barrières de protection \\
\hline
\end{tabular}

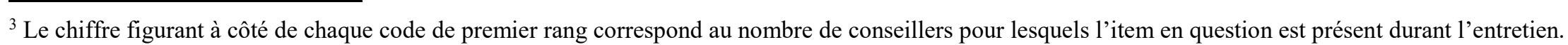




\begin{tabular}{|c|c|c|}
\hline $\begin{array}{l}\text { - Le conseiller a une formation initiale lui permettant de mieux vivre l'accompagnement - } 4 \\
\text { - Le conseiller se documente pour prendre du recul et améliorer sa posture - } 5 \\
\text { - Le conseiller reçoit des formations continues lui permettant de mieux vivre l'accompagnement - } 8 \\
\text { - Les outils/méthodes/protocoles permettent de se protéger face à des cas difficiles - } 8 \\
\text { - Les discussions / réunions de régulation avec les collègues aident à prendre du recul/analyser les pratiques - } 18 \\
\text { - Les conseillers débriefent à chaud / se soutiennent / s'entraident, de façon informelle, sur des cas difficiles - } 12 \\
\text { - La bonne ambiance au sein de l'équipe permet de mieux vivre l'accompagnement - } 4 \\
\text { - Le conseiller bénéficie (ou non) d'une supervision (ex. : soutien du supérieur hiérarchique) - } 8 \\
\text { - Le suivi par un psy / coach / un conseiller permet au conseiller de mieux vivre l'accompagnement - } 7 \\
\text { - Les accompagnements en équipe font partager la responsabilité - } 7 \\
\text { - Le conseiller transfère un cas difficile à un collègue - } 5 \\
\text { - Le conseiller se met en retrait et réoriente le bénéficiaire vers un tiers (ex. : psychologue, travailleur social) - } 17\end{array}$ & [3.2.] Ressources externes & \\
\hline $\begin{array}{l}\text { - Le tempérament du conseiller lui permet de faire face aux difficultés de l'accompagnement - } 10 \\
\text { - L'expérience professionnelle/personnelle du conseiller lui permet de mieux vivre l'accompagnement - } 14 \\
\text { - La connaissance d'un secteur / d'un public / d'un outil / d'un dispositif permet d'être plus à l'aise dans l'accompagnement - } 3 \\
\text { - La situation professionnelle du conseiller (contrat précaire, salaire ...) influe sur la manière dont il vit l'accompagnement - } 3 \\
\text { - Le conseiller disposant d'une vie personnelle stable/équilibrée vit mieux les accompagnements difficiles - } 11\end{array}$ & [3.3.] Ressources internes & \\
\hline $\begin{array}{l}\text { - Le conseiller se laisse absorber/envahir par la détresse des gens - } 8 \\
\text { - Le conseiller est attristé/peiné par l'histoire de certains bénéficiaires/éprouve de la pitié - } 9 \\
\text { - Le conseiller a un sentiment de forte responsabilité / ressent une forme de pression d'être perçu comme l'ultime recours - } 6 \\
\text { - Le conseiller est inquiet/préoccupé par la situation d'un bénéficiaire - } 4 \\
\text { - Le conseiller a peur des conséquences de ses paroles/actes (à l'extrême peur du suicide) - } 5 \\
\text { - Le conseiller donne beaucoup d'énergie dans l'accompagnement (fatigue, lassitude, usure) - } 13 \\
\text { - Le conseiller somatise (réponse physique au stress lié à l'accompagnement) - } 4 \\
\text { - Le conseiller se sent impuissant/démuni face à certains dossiers - } 12 \\
\text { - Le conseiller trouve injuste le manque de reconnaissance/l'ingratitude des bénéficiaires - } 5 \\
\text { - Le conseiller a peur pour lui-même du fait de l'accompagnement (violence physique/verbale, maladies contagieuses, etc.) - } 6 \\
\text { - Le conseiller est frustré par le manque de motivation de certains bénéficiaires - } 8 \\
\text { - Le conseiller ne souffre pas systématiquement du malheur des autres/souffre plus face à certains cas - } 3\end{array}$ & [4.1.] Contagion émotionnelle & \multirow{4}{*}{ [4.] Effets collatéraux } \\
\hline $\begin{array}{l}\text { - Le conseiller perd du sens dans son travail / le remet en cause / se démotive / abandonne - } 8 \\
\text { - Le conseiller devient dur, froid et impersonnel dans ses accompagnements - } 3 \\
\text { - Le conseiller est perturbé par des cas difficiles d'accompagnement lors d'entretiens ultérieurs - } 8 \\
\end{array}$ & $\begin{array}{l}\text { [4.2.] Désengagement } \\
\text { professionnel }\end{array}$ & \\
\hline $\begin{array}{l}\text { - Le conseiller évoque le cas de certains bénéficiaires à ses proches/ramène du travail à la maison/est moins disponible - } 11 \\
\text { - Le conseiller ne "coupe" pas / pense très souvent à certaines bénéficiaires (rêves, pensées intrusives...) - } 10 \\
\text { - Le conseiller ne s'autorise plus à vivre normalement (ex. : dépenses personnelles) / à se plaindre - } 3 \\
\text { - Le conseiller développe une vision négative de la société/est en colère contre le système/est pessimiste - } 5 \\
\text { - Le conseiller fait prendre conscience à son entourage qu'une carrière n'est pas linéaire - } 7 \\
\text { - Le conseiller devient plus vigilant/prudent dans la gestion de sa propre carrière - } 3 \\
\text { - Le conseiller a peur pour son propre avenir - } 9\end{array}$ & $\begin{array}{l}\text { [4.3.] Débordement sur } \\
\text { l'équilibre personnel }\end{array}$ & \\
\hline $\begin{array}{l}\text { - Le conseiller prend plaisir/trouve que son travail a du sens/est valorisant/utile - } 20 \\
\text { - Le conseiller se nourrit de ses rencontres (courage/persévérance de certains bénéficiaires, parcours de vie variés, etc.) - } 6 \\
\text { - Le conseiller accepte mieux ce qui lui est arrivé / relativise ses propres problèmes (santé, argent...) - } 7 \\
\text { - Le conseiller apprend à écouter les autres, y compris dans sa vie privée - } 4\end{array}$ & [4.4.] Satisfaction de compassion & \\
\hline
\end{tabular}


Une fois notre grille Gioia du traumatisme vicariant stabilisée, nous avons procédé au codage systématique, sous Word, de toutes les données recueillies (constitution d'un fichier de verbatims de 176 pages, structuré selon notre grille).

Dans la présentation des résultats empiriques (cf. infra), l'intégralité des 82 items de $1^{\text {er }}$ rang de notre grille Gioia est reprise, en structurant la partie selon les codes de $2^{\text {nd }} \operatorname{rang}^{4}$. Dans cette section «résultats empiriques», les termes en italique sont tous empruntés aux répondants, de sorte à valoriser la richesse des verbatims recueillis. Cette présentation permet finalement de dresser le « profil type » d'un conseiller en accompagnement vers l'emploi, tout en soulignant systématiquement les points faisant l'objet de certaines disparités selon les individus. Afin de préciser nos données, nous faisons figurer, dans notre grille Gioia, le nombre de répondants dont les verbatims correspondent à l'item (cf. tableau $\mathrm{N}^{\circ} 1$ ). Ce travail de comptage nous a permis d'identifier les items les plus significatifs et d'éliminer de notre analyse les caractéristiques isolées, dans le but de dresser un portrait le plus fidèle possible de la réalité d'un conseiller en accompagnement vers l'emploi.

Au final, cette démarche nous permet de générer un modèle conceptuel de la contagion de la souffrance auprès des conseillers professionnels (cf. figure $\mathrm{N}^{\circ} 1$ ). Conformément à la méthodologie 'à la Gioia', toutes les catégories de second rang issues de notre travail de codage constituent l'architecture du modèle. En définitive, il est possible d'analyser le vécu des 23 répondants et notamment la souffrance empathique liée à leur activité d'accompagnement selon le matériel traumatique auquel chacun est confronté, sa posture en tant que professionnel et ses barrières de protection. Pour «quantifier» nos données qualitatives et avoir ainsi une idée plus précise de l'ampleur du traumatisme vicariant, nous avons évalué le degré de souffrance de chaque conseiller sur une échelle de 1 à 4 (cf. tableau $\mathrm{N}^{\circ} 2$ ).

\footnotetext{
${ }^{4}$ Chaque paragraphe correspond à un thème de $2^{\text {nd }}$ rang et chaque sous-section (3.1. à 3.4.) correspond à un thème agrégé de $2^{\text {nd }}$ rang.
} 
Figure $\mathrm{N}^{\circ} 1$ : Modèle conceptuel du traumatisme vicariant des conseillers en emploi

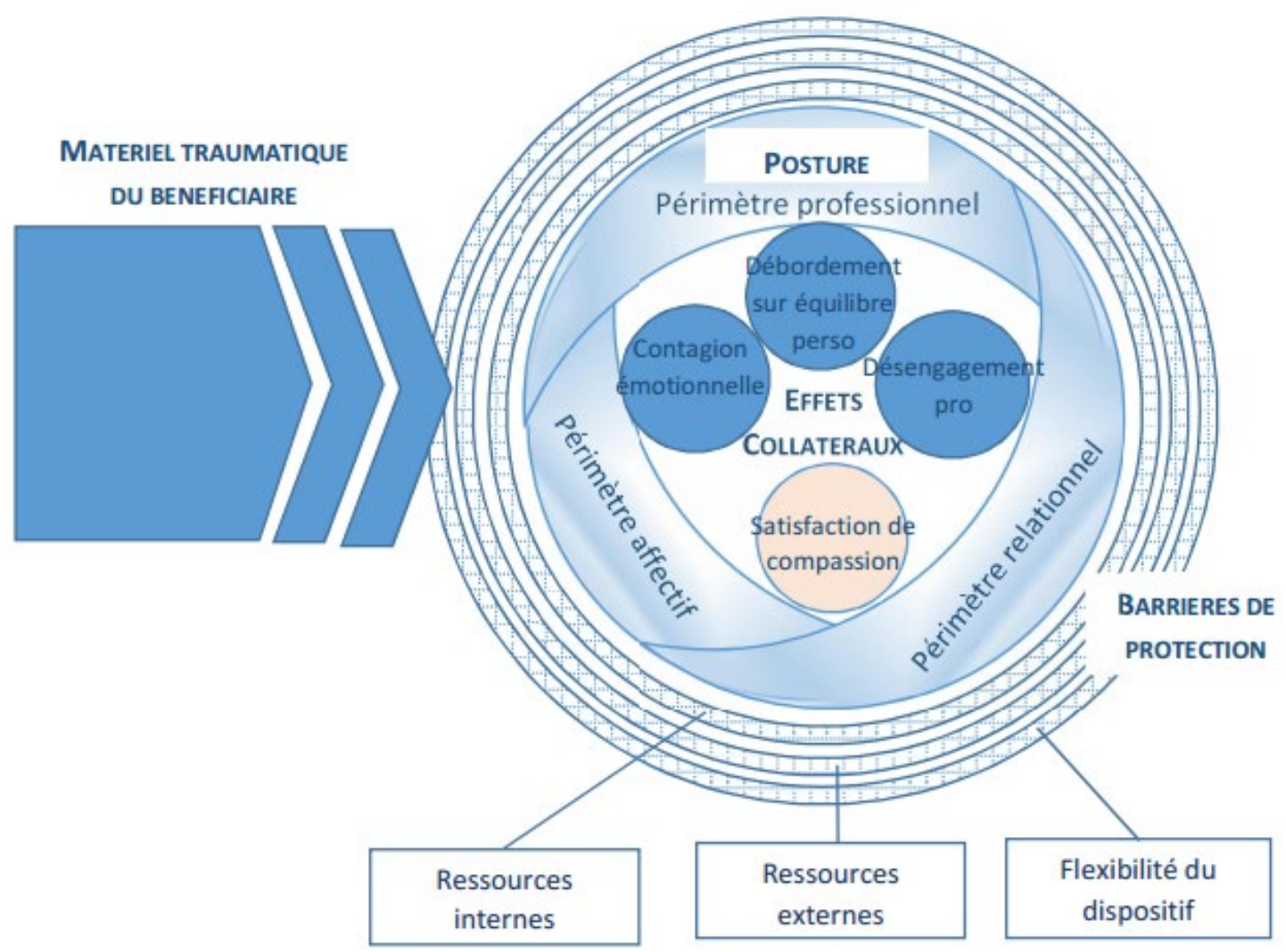

Rappelons ici que le matériel traumatique est quasiment invariant selon les conseillers, dans la mesure où nous avons conditionné le fait de sélectionner les personnes de notre échantillon au degré élevé de matériel traumatique qui leur était transmis par leurs interlocuteurs. De ce fait, nous avons analysé la contagion émotionnelle à travers deux variables seulement : la posture du conseiller et ses barrières de protection. Nous avons ensuite élaboré une typologie des conseillers en accompagnement vers l'emploi, en croisant ces deux dimensions. Plus exactement, en nous appuyant sur notre grille Gioia du traumatisme vicariant, nous avons repris les trois codes de second rang associés à ces deux dimensions ${ }^{5}$ et nous avons attribué une note de 1 à 5 à chaque conseiller sur ces aspects (cf. annexe $\mathrm{N}^{\circ} 2$ ). En calculant une moyenne pour les deux dimensions retenues, nous avons ainsi pu représenter un nuage de points dans un graphique en deux axes, dans lequel nous avons fait apparaître chaque

\footnotetext{
${ }^{5}$ Il s'agit, en l'occurrence, du périmètre professionnel, du périmètre relationnel et du périmètre affectif pour la posture, et de la flexibilité du dispositif, des ressources externes et des ressources internes pour les barrières de protection.
} 
conseiller et son degré de souffrance. Une telle typologie permet, en définitive, de voir si le modèle Gioia généré est pertinent pour interpréter chaque situation individuelle.

\section{RESULTATS EMPIRIQUES}

\subsection{MATERIEL TRAUMATIQUE DU BENEFICIAIRE}

De nombreux bénéficiaires des dispositifs d'accompagnement sont en situation de « rupture », suite à un échec professionnel (licenciement, liquidation...) parfois «violent», ayant eu des répercussions au plan social (divorce, perte de la garde des enfants, isolement, changement de statut...) et financier (fin de droits aux allocations chômage, dettes, problème de logement...). La «grande détresse » qui en résulte peut, en outre, être renforcée par des drames personnels (accident ou maladie grave, décès d'un enfant ou du conjoint, violence conjugale, prostitution...). Par ailleurs, la situation du bénéficiaire est d'autant plus difficile à vivre que celui-ci est confronté à des freins périphériques à la recherche d'emploi (handicap, absence de permis, addictions, instabilité familiale et financière, mauvaise maitrise du français...), qui réduisent ses perspectives de rebond.

Chaque parcours étant différent, l'intensité de la souffrance est variable selon les bénéficiaires. Pour les personnes les plus «abîmées », la souffrance peut se manifester de façon verbale, et ce généralement dès le premier rendez-vous avec le conseiller. De nombreux bénéficiaires expriment également, à cette occasion, leur colère - par exemple, vis-à-vis du système français d'aide au retour à l'emploi - et peuvent même se montrer «agressifs ». De même, il est assez fréquent que des personnes accompagnées fondent en larmes durant les entretiens, ce qui traduit un état «dépressif». En cas de souffrance aigüe, les individus peuvent même faire «des chantages au suicide ». La souffrance du bénéficiaire peut enfin s'observer à travers un laisser-aller général (personnes qui dorment beaucoup, alcoolisation, 
vêtements peu soignés, femmes non maquillées, hommes non rasés...), les traits de son visage, sa gestuelle (agitation, gestes compulsifs, tremblements...) ou encore son débit de parole.

Cette souffrance se répercute généralement sur les comportements du bénéficiaire. En particulier, il est fréquent que les individus «ne se mettent pas en marche » (rédaction du CV ou de la lettre de motivation, envoi de candidatures...), parce que les obstacles au retour à l'emploi leur paraissent «infranchissables ». Très souvent, leur projet professionnel «piétine », car ils «ressassent les mêmes sujets » sans progresser ou sont dans l'évitement du fait d'une «estime de soi fragilisée » (rendez-vous annulés, refus de participer à des ateliers collectifs...).

\subsection{Posture DU CONSEILler}

Confronté aux difficultés multiples des bénéficiaires, le conseiller définit le périmètre professionnel de son accompagnement. Pour certains d'entre eux, la priorité est d'être «dans l'opérationnalité », à travers la mise à disposition d'outils et de techniques d'aide à la recherche d'emploi. Certes, les conseillers prennent en compte les difficultés personnelles (famille, santé, argent, logement...) afin d'avoir une «vision globale » de la situation, mais ils ne les traitent pas eux-mêmes. Ils justifient cela en évoquant leurs «limites de compétences ». En particulier, ils refusent de jouer le rôle de psychologue ou de thérapeute. Ils peuvent ainsi être amenés à « recentrer» les échanges sur les aspects strictement professionnels durant les entretiens.

Le conseiller en emploi détermine également son périmètre relationnel avec les bénéficiaires, en fixant des règles claires dès le départ. En l'occurrence, le conseiller adopte une posture d'écoute «active », voire « empathique », et «accueille » la souffrance de ses interlocuteurs. Par ailleurs, il se montre souvent «le plus disponible» possible envers les personnes qu'il accompagne et s'adapte à leurs besoins afin d'établir une relation étroite, fondée sur la 
confiance, quitte parfois à évoquer sa propre expérience passée. La volonté affichée d'apporter de l'aide à autrui fait également souvent écho aux «valeurs » du conseiller. Néanmoins, le professionnel de l'accompagnement estime qu'il n'est pas là pour «courir après » les bénéficiaires s'ils ne sont pas suffisamment «impliqués ». L'objectif est de les «responsabiliser » et de les rendre «autonomes » dans la démarche de retour à l'emploi. De plus, le conseiller garde une certaine distance avec les individus accompagnés (vouvoiement, pas d'intrusion dans la vie privée, pas de familiarité, pas de relation privée...).

Enfin, le conseiller en emploi établit le périmètre affectif de son accompagnement. Le maîtremot ici est de «prendre du recul », au plan émotionnel, par rapport aux situations vécues par les bénéficiaires. Selon cette vision, le meilleur moyen d'aider les individus en souffrance est d'éviter de tomber dans la compassion. Le conseiller ne souhaite pas non plus être perçu comme un «sauveur » pour ne surtout pas créer un "sentiment de dépendance ». Idéalement, le conseiller intègre d'emblée le fait qu'il ne parviendra pas systématiquement à être «utile » aux personnes en recherche d'emploi. Malgré leur volonté déclarée de se «détacher», beaucoup d'accompagnants admettent toutefois que le «dosage» entre empathie et distance est difficile à trouver. D’ailleurs, ils reconnaissent généralement qu'ils n'ont pas le même ressenti selon les bénéficiaires. Ils peuvent notamment avoir "plus d'affinités » avec des personnes particulièrement «courageuses » et «motivées », tout comme ils peuvent se sentir plus proches - par « effet miroir »-d'individus ayant traversé les mêmes épreuves qu'eux.

\subsection{BARRIERES DE PROTECTION}

Premièrement, l'accompagnement de personnes en souffrance est mieux vécu par le conseiller lorsque le dispositif dont il dispose offre une certaine «souplesse » et bénéficie de moyens importants. Les conseillers préfèrent ainsi avoir une "marge de liberté » en termes de durée des entretiens ou du suivi, car cela leur donne le sentiment de faire un travail «de qualité » et de voir les personnes «évoluer». A l'inverse, certains conseillers ont du mal à s'épanouir 
dans un contexte où on leur met la "pression » pour être «rentable » et où ils doivent « rendre des comptes » par rapport à des indicateurs (insertion professionnelle, placement en formation, prestation...). C'est le cas principalement quand ils doivent gérer d'importants portefeuilles de bénéficiaires, qu'ils reçoivent «à la chaîne », dans un temps très limité, car ils ont conscience qu'une telle «cadence» nuit à l'accompagnement. De plus, une forte «fréquence d'exposition » à des personnes traumatisées rend le travail de conseiller difficile à supporter, du fait justement de cette confrontation «répétée » à la souffrance humaine. À ce titre, il apparaît que les conseillers polyvalents - i.e. ne faisant pas uniquement des entretiens avec des demandeurs d'emploi (prospection ou conseils aux entreprises, tâches administratives, réponse à des appels d'offres...) - vivent mieux l'accompagnement. Leurs autres activités font office de «soupapes » de décompression et leur offrent la possibilité de «digérer » les émotions négatives. De la même façon, le fait d'entrevoir des "possibilités d'évolution » professionnelles est réconfortant pour le conseiller, car il perçoit souvent son métier comme ne pouvant être que «temporaire ».

Deuxièmement, des ressources externes sont mobilisées par le conseiller pour se protéger face à la souffrance des bénéficiaires. Une formation initiale en psychologie, par exemple, donne des repères et aide le conseiller à trouver sa posture. De même, des formations continues régulières (gestion du stress, effets miroirs, gestion du temps, relation avec les publics difficiles...) permettent de «prendre de la hauteur». En outre, le fait de pouvoir se reposer sur des méthodes et techniques concrètes d'accompagnement (ex.: déroulement des entretiens) se révèle souvent rassurant. Plusieurs conseillers évoquent aussi leur effort constant de documentation pour réfléchir à leur posture. Surtout, face au risque de contamination des émotions, les professionnels de l'accompagnement sont unanimes pour souligner l'importance des «réunions de régulation». Discuter de leurs dossiers les plus difficiles avec leurs pairs les aident à prendre du recul, car ces derniers peuvent avoir un 
«regard différent», voire «apporter une solution». Ces «inter-visions» permettent d'analyser des situations, d'échanger des pratiques et de ne pas se sentir «seul». Plus généralement, «l'entraide» au sein d'une équipe «soudée », où règne une «bonne ambiance », ressort comme un élément clef aux yeux des conseillers face au risque de traumatisme vicariant. Parfois, les accompagnements se font en équipe (binôme conseiller/psychologue), ce qui permet de partager la responsabilité de l'accompagnement. Le conseiller peut aussi «transférer » un cas difficile à un autre collègue lorsqu'il a le sentiment d'être dans une «impasse » et de «se fragiliser ». De même, quand le suivi est entravé par les problématiques personnelles du bénéficiaire, le conseiller peut se «mettre en retrait» et «réorienter» le bénéficiaire vers un tiers (psychologue/psychothérapeute, travailleur social...). L'existence d'une «supervision» avec un supérieur hiérarchique "à l'écoute» constitue une autre barrière de protection importante. Il est particulièrement apprécié que celui-ci connaisse les spécificités du métier de conseiller, puisse apporter une aide sur les dossiers et comprendre ce que ressent le conseiller face à des cas complexes. Certains conseillers éprouvent également le besoin d'être accompagnés par un psychologue ou un coach extérieur. Outre l'évacuation des émotions négatives, ce choix s'inscrit dans une démarche de « développement personnel ».

Troisièmement, confronté au matériel traumatique des personnes qu'il accompagne, le conseiller peut s'appuyer sur des ressources internes. Ainsi, son tempérament (optimisme, pragmatisme, caractère fort, résistance, distance naturelle, capacité à relativiser...) semble avoir une influence considérable sur son ressenti. De même, l'expérience et la maturité aident, au fil du temps, à être plus «serein» face à des personnes en grande souffrance et à «se détacher ». Les connaissances détenues par le conseiller (sur le public accompagné, le secteur d'activité visé, les outils à disposition...) constituent une autre ressource permettant de mieux vivre l'accompagnement. La stabilité de la situation du conseiller entre également en ligne de 
compte. Ainsi, le conseiller est d'autant plus facilement fragilisé par l'accompagnement qu'il est lui-même dans une situation professionnelle précaire (contrat, salaire, incertitude quant à l'avenir...). En parallèle, pouvoir compter sur une vie privée "équilibrante » (entourage, logement, santé, argent, loisirs...) est un socle précieux, car cela lui offre des possibilités de « décompresser ».

\subsection{EFFETS COLLATERAUX}

Malgré toutes les barrières de protection existantes, le conseiller n'est «pas insensible ». En effet, les bénéficiaires sont parfois source d'anxiété (colère et insultes durant les entretiens, courriels incendiaires, menaces...). De plus, les conseillers sont victimes d'une certaine « ingratitude » de la part de personnes auxquelles ils viennent en aide, ce qui peut développer un «sentiment d'injustice ». Enfin, ils sont «peinés » par la situation de certains publics très fragilisés. Le conseiller reçoit parfois « de plein fouet» la souffrance des bénéficiaires. Leur situation difficile peut le «préoccuper », jusqu'à ressentir une forte «pression » s'il a le sentiment d'être l'ultime recours, notamment vis-à-vis de personnes pour lesquelles il pressent un risque de suicide. Certains, dans des cas extrêmes, se laissent littéralement «submerger » par la détresse des gens. Il peut aussi arriver que les conseillers se sentent « impuissants » et «démunis » face aux dossiers dont ils ont la charge, compte tenu des «leviers d'action » à leur disposition. Plus généralement, les conseillers ont le sentiment de donner « beaucoup d'énergie » dans l'accompagnement des publics fragiles et ressentent très souvent de la «fatigue» (physique et psychique). Cette «usure » est d'autant plus pesante que les professionnels de l'accompagnement sont confrontés à des individus manquant parfois de motivation, ce qui est source de «frustration », voire d'agacement. En définitive, tout cela peut avoir des impacts négatifs sur la santé des conseillers et se traduire par des arrêts maladie. 
Au-delà de cette contagion émotionnelle, le conseiller peut aussi se désengager professionnellement au fil du temps. Ainsi, le professionnel perd parfois du «sens» dans son travail. Dans ce cas-là, il peut envisager de «changer de métier » ou alors il devient «dur », «froid», « impersonnel », voire «violent » dans ses accompagnements. Le conseiller peut aussi être perturbé du fait d'un entretien difficile dans ses rendez-vous suivants (déconcentration, manque de recul, perte de dynamisme, écoute insuffisante...).

Au-delà des conséquences professionnelles, le conseiller peut aussi être victime de débordements sur son équilibre personnel. En particulier, il peut évoquer le cas de certains bénéficiaires à ses proches contre leur gré, ramener du travail à son domicile ou encore avoir des «pensées » intrusives en lien avec l'accompagnement (y compris la nuit), ce qui traduit alors une difficulté à «couper ». Il arrive même que le conseiller change son comportement au quotidien, notamment lorsqu'il «ne s'autorise plus » à vivre normalement (ex. : dépenses personnelles jugées déplacées au regard de l'indigence des bénéficiaires). En rencontrant des publics en souffrance, les conseillers modifient aussi leurs représentations. Ils peuvent notamment estimer qu'ils n'ont «pas le droit de se plaindre » de leurs problèmes personnels. Ils développent parfois une «vision négative » de la société (management dans les entreprises, politique publique...), au point d'être «en colère ». De même, la nature du métier peut conduire les conseillers à appréhender différemment leur avenir professionnel ou celui de leurs proches. Certains d'entre eux sont inquiets, voire «angoissés ». La plupart développent, peu à peu, le sentiment que «personne n'est épargné», que l'on peut perdre son travail du jour au lendemain et que le contexte actuel est «dur». Dès lors, ils peuvent devenir plus prudents dans la gestion de leur carrière. Leurs enfants sont souvent sensibilisés à l'importance de l'employabilité et au fait qu'une carrière n'est pas «linéaire ».

Toutefois, il apparaît aussi qu'un accompagnement bien vécu peut avoir des effets positifs. Les conseillers sont ainsi unanimes pour souligner le «plaisir» qu'ils ont de «se sentir 
utiles » lorsqu'ils ont contribué à la reprise de confiance et au retour à l'emploi d'une personne. Leur travail de «catalyseur» est «valorisant» à leurs yeux. En outre, les professionnels de l'accompagnement soulignent qu'ils «se nourrissent» de leurs rencontres (courage et persévérance de certains bénéficiaires, parcours de vie extrêmement variés...), car celles-ci sont sources d'apprentissage (regard différent sur la vie, découverte d'autres cultures...). Ce vécu par procuration leur permet de relativiser lorsque leurs problèmes personnels sont relativement mineurs (ex. : petits soucis de santé, budget insuffisant pour partir en vacances, difficultés à financer les études des enfants, problèmes matériels du quotidien...). Ils prennent conscience que leur situation n'est pas si «dramatique » et profitent des «petits bonheurs du quotidien ». Enfin, à travers sa posture empathique vis-à-vis des bénéficiaires en souffrance, le conseiller développe une capacité d'écoute, dont il tire profit dans sa vie privée et qui lui est souvent reconnue par son entourage.

\subsection{APPLICATION DU MODELE POUR LA CONSTRUCTION D'UNE TYPOLOGIE}

Les différents éléments issus du modèle (cf. figure $\mathrm{N}^{\circ} 1$ et sections 3.1. à 3.4.) recensent l'ensemble des ressentis et vécus des accompagnants. Lorsque l'on affine les résultats par une analyse individuelle, il apparaît clairement que la souffrance expérimentée diffère selon les conseillers. Ainsi, nous avons défini quatre niveaux de contagion vis-à-vis de la souffrance du bénéficiaire (cf. tableau $\mathrm{N}^{\circ}$ 2). Bien que la souffrance soit présente chez la quasi-totalité des conseillers, on observe l'existence d'un «point de bascule » liée à la permanence de cette souffrance. Ainsi, pour la catégorie 2, la souffrance se limite au moment de l'interaction avec le bénéficiaire : ces conseillers n'ont pas de mal à y faire face. En revanche, pour les catégories 3 et 4 , la souffrance perdure parfois au-delà de l'entretien, de la sphère professionnelle et devient préoccupante. 
Tableau $\mathrm{N}^{\circ} 2$ : Échelle de souffrance du conseiller et effectifs affectés à chaque catégorie

\begin{tabular}{|l|c|}
\hline & Effectifs \\
\hline $1:$ Cela ne me touche pas de manière significative. & 1 \\
\hline 2 : Certains cas me touchent de façon significative pendant l'entretien mais pas au-delà. & 12 \\
\hline $\mathbf{3}$ : Certains cas me touchent de façon significative plusieurs jours après l'entretien. & $\mathbf{6}$ \\
\hline $\mathbf{4}:$ Certains cas me touchent au point de me laisser envahir. & $\mathbf{4}$ \\
\hline
\end{tabular}

De plus, les résultats généraux montrent que s'ils font tous face à de la grande souffrance, les conseillers n'adoptent pas tous la même posture et ne disposent pas des mêmes barrières de protection pour se prémunir. La typologie présentée ci-dessous (cf. figure $\mathrm{N}^{\circ} 2$ ) a donc pour objectif de lire notre modèle Gioia du traumatisme vicariant (cf. figure $\mathrm{N}^{\circ} 1$ ) individu par individu. Elle consiste en une représentation graphique de la situation de chaque individu, en fonction de sa posture, de ses barrières de protection et de son degré de souffrance.

En l'occurrence, notre typologie montre que les conseillers apparaissant dans les zones les moins protégées (c'est-à-dire dans les cadrans $n^{\circ} 2,3$ et 4 de la figure) obtiennent tous une note de souffrance élevée (de 3 ou 4$)^{6}$. Il apparait ainsi que le traumatisme vicariant est bien dû à un problème de posture du conseiller et/ou à un manque de barrières de protection. À l'inverse, les conseillers qui ne sont pas victimes de contamination émotionnelle apparaissent tous dans le cadran correspondant à une posture contrôlée et à des barrières de protection fortes (cf. cadran $\mathrm{N}^{\circ} 1$ ).

\footnotetext{
${ }^{6}$ Les lettres de $\mathrm{A}$ à $\mathrm{W}$ représentent les 23 conseillers. Le chiffre accolé à chaque lettre correspond, quant à lui, au degré de souffrance de chaque conseiller.
} 
Figure $\mathrm{N}^{\circ} 2$ : Typologie des conseillers

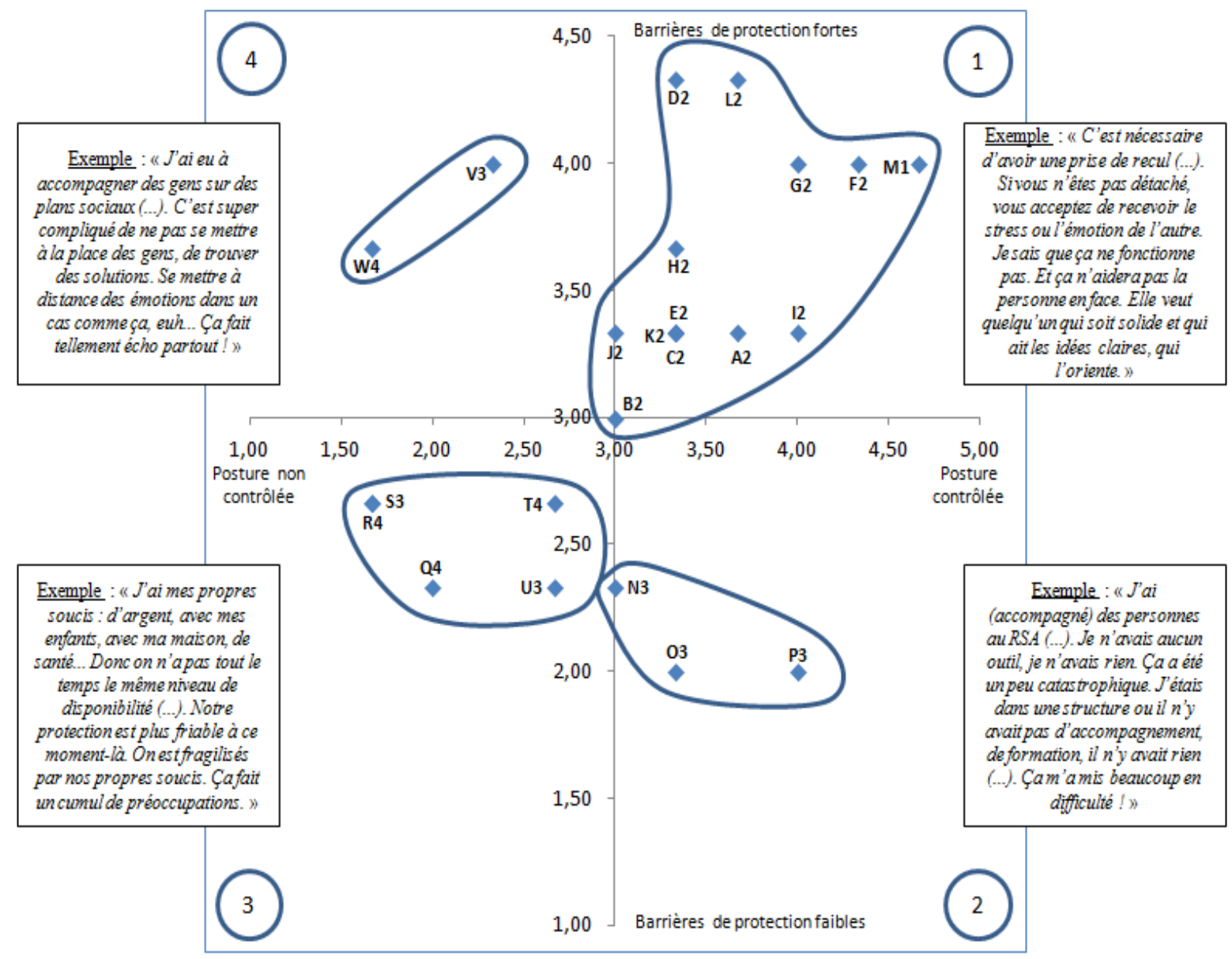

\section{DISCUSSION}

\subsection{DISCUSSION DU MODELE CONCEPTUEL}

Dans cet article, nous nous sommes appuyés sur le concept de traumatisme vicariant (McCann et Pearlman, 1990 ; Pearlman et Saakvitne, 1995), emprunté à la littérature médicale, pour construire un modèle conceptuel de la souffrance au travail par contagion empathique. Ce modèle, synthétisé dans la figure $\mathrm{N}^{\circ} 1$, constitue le principal résultat de cette recherche. En effet, en partant des données émanant du terrain pour construire la première étape de notre grille 'Gioia' (le codage de premier rang), nous avons retrouvé sans difficulté de nombreux éléments ayant trait au matériel traumatique, aux facteurs de risques et aux effets collatéraux, tels que cités dans la littérature sur le traumatisme vicariant. Il apparait, dès lors, que ce concept s'ajuste particulièrement bien aux phénomènes observés sur le terrain de l'accompagnement professionnel, et que l'intégration d'un tel concept dans le champ de la 
recherche en gestion des ressources humaines a du sens. Mieux, cette transposition du traumatisme vicariant permet d'enrichir son étude, en intégrant au modèle conceptuel des variables relativement délaissées par la médecine, mais centrales pour la gestion des RH. Par exemple, la posture de l'accompagnant n'est pas prise en compte dans les recherches en médecine, qui se focalisent plus naturellement sur l'étude des facteurs de risque du traumatisme vicariant et de ses symptômes. A l'inverse, il apparaît que la distinction médicale - qui reste floue - entre fatigue de compassion et traumatisme vicariant (Sabin-Farrell et Turpin, 2003) n'a pas forcément lieu d'être dans une perspective RH. Au-delà de la nature de l'effet collatéral (cognitif versus comportemental), ce qui importe réellement au plan RH est d'identifier les conditions d'existence d'une souffrance au travail liée à l'accompagnement de publics traumatisés, et les pratiques RH qui en limitent les risques. Cette transposition réussie et ces adaptations nous conduisent, finalement, à proposer une définition RH du traumatisme vicariant: «Dans les métiers supposant un engagement empathique fort, c'est l'effet collatéral négatif durable d'une exposition prolongée et/ou répétée à une souffrance aiguë exprimée par les bénéficiaires, dont l'intensité varie selon la posture et les barrières de protection du professionnel. »

Un autre apport conceptuel est lié à l'analyse d'un type bien particulier de souffrance au travail, due à l'empathie ressentie à l'égard d'interlocuteurs eux-mêmes en souffrance. Nos résultats permettent de mettre en exergue son existence, de mieux comprendre les conditions de son émergence et les mécanismes de sa gestion individuelle. Plus précisément, nous montrons que l'existence (ou non) d'un traumatisme vicariant dépend de trois variables clefs : le matériel traumatique, la posture du conseiller et les barrières de protection. Vu que tous les conseillers interrogés sont confrontés à un matériel traumatique extrêmement lourd (du fait même de la construction de notre échantillon), il en ressort que la forte disparité observée au niveau des effets collatéraux négatifs (cf. tableau $\mathrm{N}^{\circ} 2$ ) est à relier aux différences de posture 
et/ou de barrières de protection individuelles. À travers notre typologie, les résultats montrent que des histoires dissemblables peuvent toutes se lire à l'aune du modèle théorique proposé.

\subsection{DISCUSSION AUTOUR DES DIFFERENTES VARIABLES DU MODELE CONCEPTUEL}

Concernant chacune des quatre variables composant le modèle, nos résultats, d'une part, confirment certains résultats déjà connus et d'autre part, permettent d'éclairer le concept de traumatisme vicariant ainsi que la littérature sur la souffrance au travail sous un jour nouveau. Tout d'abord, en identifiant des effets collatéraux, tels que la contagion émotionnelle, le désengagement professionnel et le débordement sur l'équilibre personnel, nous confirmons ici l'idée selon laquelle les métiers du «care » sont susceptibles de provoquer une souffrance indirecte (Beck, 2011 ; Figley, 1995 ; Ide, 2015 ; Jenkins et al., 2011 ; Maslach, 1982 ; Shiri et al., 2010 ; Sinclair et Hamill, 2007 ; Trippany et al., 2004 ; Way et al., 2004). En effet, selon notre classification, seul un répondant (sur un total de 23) semble totalement détaché par rapport aux récits douloureux des bénéficiaires. Pour autant, nous avons vu que les conseillers ne sont pas tous touchés de la même façon par la souffrance de leurs interlocuteurs. Nous faisons ainsi apparaitre un «point de bascule » liée à la rémanence des effets collatéraux négatifs. Selon cette perspective, on peut réellement parler de traumatisme vicariant si le trouble du conseiller persiste quelque temps après que l'interaction avec le bénéficiaire a eu lieu. En parallèle, notre recherche confirme qu'aux côtés des dimensions négatives liées à l'accompagnement professionnel de publics en souffrance coexistent de nombreux aspects positifs pour les conseillers (Mangoulia et al., 2015 ; Sinclair et Hamill, 2007). La littérature reste toutefois évasive sur cette satisfaction de compassion et notre article permet d'en définir plus clairement les contours. Ainsi, celle-ci va au-delà du sentiment d'utilité, traditionnellement évoqué par les auteurs. À titre d'illustration, en écho à l'importance des changements cognitifs à long terme, propres au traumatisme vicariant (Elwood et al., 2011 ; 
Pearlman et Saakvitne, 1995 ; Sabin-Farrell et Turpin, 2003 ; Shiri et al., 2010 ; Sinclair et Hamill, 2007 ; Way et al., 2004), nous montrons que l'accompagnement de publics fragiles peut changer les schémas cognitifs de façon positive (ex. : volonté de profiter de la vie).

Par ailleurs, les barrières de protection, issues de notre travail de catégorisation des données du terrain, correspondent en creux aux facteurs de risque du traumatisme vicariant, repérés dans la littérature médicale. Il s'agit là, en effet, des deux faces d'une même pièce. Nous retrouvons ainsi un certain nombre de facteurs déjà identifiés dans la littérature, tels que l'expérience (Bober et Regehr, 2006 ; Meyers et Cornille, 2002), le contexte de vie actuel (Saakvitne et Pearlman, 1996), l'accès à la formation (Eriksen et Ditrich, 2015 ; Vrklevski et Franklin, 2008 ; Way et al., 2004), l'histoire personnelle (Devilly et al., 2009 ; Saakvitne et Pearlman, 1996), le soutien des pairs (Sinclair et Hamill, 2007 ; Trippany et al., 2004) ou encore la supervision (Sinclair et Hamill, 2007 ; Vrklevski et Franklin, 2008 ; Way et al., 2004). En revanche, parmi tous les facteurs de risque repérés dans la littérature médicale, et notamment par Pearlman et Saakvitne (1995), deux n'ont pas du tout été évoqués spontanément par nos répondants, à savoir la spiritualité et le genre ${ }^{7}$. Surtout, nos résultats identifient de nouvelles barrières de protection pour le conseiller, telles que sa maîtrise du sujet (public, secteur, dispositif, etc.) ou la possibilité de réaliser les accompagnements en équipe, de transférer un cas difficile à un collègue et de réorienter le bénéficiaire vers un tiers. Nos résultats soulignent également l'importance du degré de flexibilité du dispositif entre les mains du conseiller. Ils donnent notamment à voir de nouveaux facteurs de risques, qui concernent la gestion des ressources humaines au premier chef, à savoir les contraintes de rentabilité pesant sur les conseillers, le manque de polyvalence du poste et l'absence de perspectives d'évolution professionnelle. On retrouve ici certains facteurs identifiés par

\footnotetext{
${ }^{7}$ Notons toutefois ici que les quatre conseillers situés les plus en haut à droite de notre typologie (cf. figure $\mathrm{N}^{\circ} 2$ ) sont tous des hommes (sur un total de huit répondants du même sexe). De même, tous les hommes obtiennent une note de 4 ou 5 pour les ressources internes, alors que la moyenne générale pour ce code est de 3,6. Cela laisse supposer qu'une analyse en termes de genre pourrait être intéressante pour des recherches futures.
} 
Karasek (1979) comme des remparts face au stress : le soutien social, l'autonomie ou les ressources en termes de compétences et de formation notamment.

Les caractéristiques personnelles (genre, âge...) ou contextuelles (degré d'exposition au traumatisme, accès à la supervision, mise en place de formation, soutien social...) habituellement étudiées (Pearlman et Mac Ian, 1995 ; Pearlman et Saakvitne, 1995) sont généralement en-dehors du contrôle de l'individu. A contrecourant de cette représentation implicite, nos résultats établissent que le conseiller ne reste pas passif dans sa confrontation à la souffrance de ses interlocuteurs. En effet, celui-ci adopte une posture particulière vis-à-vis du matériel traumatique. Nos résultats montrent, de plus, que cette posture peut se définir à l'aune de trois périmètres : professionnel, relationnel et affectif. Pour chacun d'entre eux, le conseiller positionne un «curseur », qui lui est propre. À titre d'illustration, concernant le périmètre professionnel, la dimension systémique du matériel traumatique - liée aux difficultés économiques et sociales, venant aggraver la souffrance du bénéficiaire - suppose que le conseiller délimite sa zone de compétences (ce qu'il peut traiter vs. ce qu'il ne peut pas traiter), au-delà de laquelle il estime qu'il se mettra personnellement en danger. L'enjeu de la définition du périmètre est d'autant plus crucial que le rôle du conseiller peut paraître flou aux yeux des bénéficiaires. Ces derniers peuvent donc voir en leur conseiller un "sauveur», capable de tout prendre en charge, et l'amener - même inconsciemment - sur des terrains où il n'a pas vocation à intervenir.

\subsection{DISCUSSION AUTOUR DE LA METHODOLOGIE DE LA RECHERCHE}

De notre point de vue, le modèle revêt également un intérêt méthodologique. En effet, il a été conçu en mobilisant la démarche de codification préconisée par Gioia et al. (2013). Si cet article-là est de plus en plus cité dans les meilleures revues scientifiques à l'échelle internationale (à titre d'exemple, en février 2016, l'article de Gioia et al. (2013) avait déjà été 
utilisé dans sept articles publiés dans Academy of Management Journal), il reste encore ignoré des revues en GRH. C'est le cas également de l'article fondateur de la méthodologie dite 'à la Gioia', publié par Corley et Gioia (2004) dans Administrative Science Quarterly. Parmi toutes les revues scientifiques en GRH classées par le CNRS et disponibles sur Business Source Premier, Cairn, Sage et Science Direct, il semblerait que seul l'article de Loufrani-Fedida et al. (2015: p.26), publié dans la Revue de Gestion des Ressources Humaines, fait référence dans sa méthodologie à l'un des deux travaux mentionnés supra ${ }^{8}$. En outre, cet article parle d'une «structuration des données (...) inspiré(e) de Corley et Gioia (2004) », sans trop s'étendre sur les spécificités de cette démarche méthodologique. Le présent article, à l'inverse, donne à voir l'intérieur de la «boîte noire » du codage, à partir duquel le modèle a été élaboré. Dès lors, nous pouvons imaginer que notre approche, résumée par le tableau $\mathrm{N}^{\circ}$, serve de base à de futures recherches qualitatives en GRH. Par ailleurs, en lien plus spécifiquement avec le sujet que nous étudions ici, il apparaît que notre 'grille Gioia du traumatisme vicariant' constitue un premier pas en vue d'opérationnaliser puis de mesurer les concepts clefs associés au traumatisme vicariant (matériel traumatique, posture, barrière de protection et effets collatéraux) et de tester le modèle d'un point de vue quantitatif.

\subsection{LIMITES, PISTE DE RECHERCHE ET IMPLICATIONS MANAGERIALES}

L'une des limites de ce travail concerne justement la validité externe du modèle, qui peut être interrogée au regard de la taille réduite de notre échantillon. Il convient toutefois de rappeler que le recueil des données n'a été interrompu qu'après avoir atteint le seuil de saturation sémantique. Une autre limite de notre recherche est liée au spectre très large de structures étudiées, si bien qu'un ou deux répondants seulement par structure ont généralement été

\footnotetext{
${ }^{8}$ L'article de Ng et Roberts (2007), publié dans Human Relations, cite certes Corley et Gioia (2004) dans la méthodologie, mais les auteurs ne mobilisent pas la méthode de codage de premier rang et de second rang. En revanche, dans cette même revue, Corley (2004) mobilise clairement cette démarche, sans toutefois faire référence à l'article qu'il a coécrit, la même année, dans Administrative Science Quarterly.
} 
interrogés. En d'autres termes, nous n'avons approfondi aucun contexte particulier. Dès lors, une piste de recherche possible consisterait à changer d'unité d'analyse, en se focalisant cette fois-ci non plus sur les conseillers, mais sur les structures d'accompagnement afin d'opérer des comparaisons inter-cas entre deux ou trois structures particulières.

Nonobstant ces limites, notre étude apporte des éléments de solutions préventives aux organisations et aux individus qui évoluent dans des secteurs d'activités propices au traumatisme vicariant. En particulier, en soulignant le rôle du conseiller et des trois périmètres dans lequel il inscrit sa posture, nous invitons les professionnels du secteur à préciser leur posture et à la surveiller, et leur superviseur à les accompagner dans ce processus. Ainsi, concernant le périmètre professionnel, il semble important que la structure clarifie le rôle de l'accompagnant, en le formant, en lui donnant des repères, pour que la personne sache qu'elle ne peut pas gérer toutes les problématiques de son interlocuteur, pour qu'elle identifie bien ce qui fait partie de sa mission et ce qui n'en relève pas. Ainsi, l'accompagnant pourra éviter la confusion entre son rôle et celui d'un psychologue, par exemple. Le périmètre relationnel concerne, quant à lui, l'échange et les responsabilités mutuelles dans la relation. Il semble ici important que le conseiller ait bien à l'esprit qu'il «tient conseil» plus qu'il ne le donne, c'est-à-dire que la solution ne dépend pas de lui, mais de la capacité de l'interlocuteur à se saisir de l'aide qui lui est proposée pour trouver sa propre solution. Ces précautions permettront ainsi au conseiller de ne pas se vivre comme un «sauveur », et de ne pas endosser la responsabilité de la réussite ou de l'échec de la trajectoire (parfois dramatique) de la personne.

Ce travail sur la posture peut être mené individuellement, par des lectures théoriques, par une prise de recul régulière sur sa pratique, ou par le fait de suivre soi-même une thérapie de façon à conscientiser les risques encourus et de mieux s'en prémunir. Cependant, il parait nécessaire d'insister sur la capacité des organismes d'aide à l'insertion professionnelle à protéger leurs 
salariés ou leurs bénévoles. Ainsi, toutes les pratiques permettant d'interroger régulièrement la pratique du métier offrent une aide précieuse aux salariés : réunions hebdomadaires d'échanges de pratiques entre pairs (séances d'inter-visions), rencontres collectives régulières avec un psychologue afin de pouvoir être écouté au sujet des difficultés rencontrées, soutien d'un superviseur, apportant une aide à la fois technique, morale et sécurisante (supervision), formations régulières autour de la posture de conseiller en insertion professionnelle, etc. D'autres conditions de travail paraissent essentielles pour pouvoir se ressourcer : on retiendra principalement le fait d'avoir des pauses entre les différents entretiens, qui servent de « soupapes » de décompression, le fait de pouvoir aller discuter avec des collègues lorsque le besoin s'en fait ressentir ou encore le fait d'avoir de la marge de manœuvre dans le dispositif à suivre (choix des personnes à rencontrer en rendez-vous, du rythme de rencontres, du temps imparti à chaque rencontre, etc.). 


\section{BIBLIOGRAPHIE}

ALIS D. (2010) « Le travail émotionnel des salariés en contact avec le public : Prévenir les risques de dissonance », XXIème congrès de l'AGRH, Rennes Saint-Malo, 17-19 novembre. AQUILANTI T. et LEROUX J. (1999) «An Integrated Model of Outplacement Counseling », Journal of Employment Counseling, Vol. 36, p. 177-192.

BALZANI B., BERAUD M., BOULAYOUNE A., DIVAY S., EYDOUX A. et GOUZIEN A. (2008) «L'accompagnement vers l'emploi. Acteurs, pratiques, dynamiques », Rapport de recherche pour la DARES, p. 1-273.

BECK C. (2011) «Secondary Traumatic Stress in Nurses: A Systematic Review », Archives of Psychiatric Nursing, Vol. 25, $\mathrm{N}^{\circ} 1$, p. 1-10.

BEN-ZUR H. et YAGIL D. (2005) «The relationship between empowerment, aggressive behaviours of customers, coping, and burnout », European Journal of Work \& Organizational Psychology, Vol. 14, N¹, p. 81-99.

BOBER T. et REGEHR C. (2006) «Strategies for reducing secondary or vicarious trauma: Do they work? », Brief Treatment and Crisis Intervention, Vol. 6, N¹, p. 1-9.

BRESMAN H. (2013) «Changing Routines: A Process Model of Vicarious Group Learning in Pharmaceutical R\&D », Academy of Management Journal, Vol. 56, N¹, p. 35-61.

BUE J., COUTROT T., GUIGNON N. et SANDRET N. (2008) « Les facteurs de risques psychosociaux au travail. Une approche quantitative par l'enquête Sumer », Revue française des affaires sociales, Vol. 2, p. 45-70.

CADIN L., BENDER A.-F. et DE SAINT GINIEZ F. (2003) Carrières nomades : les leçons d'une comparaison international, Paris : Vuibert.

CHOI G.-Y. (2011) «Organizational Impacts on the Secondary Traumatic Stress of Social Workers Assisting Family Violence or Sexual Assault Survivors ", Administration in Social Work, Vol. 35, p. 225-242.

CLOT, Y. (1995) Le travail sans l'homme? Pour une psychologie des milieux de travail et de vie. Paris : La Découverte.

CLOT Y. (2008). Travail et pouvoir d'agir. Le travail humain, Paris: PUF.

CLOT (2010) Le Travail à cour. Pour en finir avec les risques psychosociaux. Paris: La Découverte.

CORDES C. et DOUGHERTY T. (1993) «A review and an integration of research on job burnout ", Academy of Management Review, Vol. 18, p. 621-656.

CORLEY K. (2004) «Defined by our strategy or our culture? Hierarchical differences in perceptions of organizational identity and change », Human Relations, Vol. 57, p. 1145-1177.

CORLEY K. et GIOIA D. (2004) «Identity ambiguity and change in the wake of a corporate spin-off », Administrative Science Quarterly, Vol. 49, p. 173-208.

DAY H. et CHAMBERS J. (1991) «Empathy and burnout in rehabilitation counsellors», Canadian Journal of Rehabilitation, Vol. 5, N¹, p. 33-44.

DANY F. (1997) La promesse d'employabilité : un substitut possible à la promesse de carrière? Thèse de doctorat, Université Jean Moulin Lyon III.

DEBOUT M. (1999). Travail, violences et environnement. Avis adopté par le Conseil Economique et Social lors de la séance du 24 octobre 1999.

DEJOURS (1998) : Souffrance en France : La banalisation de l'injustice sociale, Paris, Seuil. DEJOURS C. (sous la direction de) (2007), Conjurer la violence. Travail, violence et santé, Paris : Payot.

DEJOURS C. (2010) Observations cliniques en psychopathologie du travail, Paris : PUF.

DEMEROUTI E., BAKKER A., NACHREINER F. et SCHAFUELI W. (2001) « The job demands-resources model of burnout ", Journal of Applied Psychology, Vol. 86, p. 499-512. 
DEVILLY G., WRIGHT R. et VARKER T. (2009) «Vicarious trauma, secondary traumatic stress or simply burnout? Effect of trauma therapy on mental health professionals ", Australian and New Zealand Journal of Psychiatry, Vol. 43, p. 373-385.

ELWOOD L., MOTT J., LOHR J., et GALOVSKI T. (2011) « Secondary trauma symptoms in clinicians: A critical review of the construct, specificity, and implications for traumafocused treatment », Clinical Psychology Review, Vol. 31, p. 25-36.

ERIKSEN C. et DITRICH T. (2015) « The relevance of mindfulness practice for traumaexposed disaster researchers ", Emotion, Space and Society, Vol. 17, p. 63-69.

FIGLEY C. (1995) "Compassion fatigue as secondary traumatic stress disorder: an overview ", In STAMM B. (Ed.) Compassion fatigue: coping with secondary traumatic stress disorder in those who treat the traumatized, New York: Brunner-Mazel, p. 1-20.

GENTRY J., BARANOWSKY A. et DUNNING K. (2002) "ARP: The Accelerated Recovery Program (ARP) for compassion fatigue », In FIGLEY C. (Ed.) Treating compassion fatigue, New York: Brunner-Rutledge, p. 123-137.

GIOIA D., CORLEY K. et HAMILTON A. (2013) « Seeking Qualitative Rigor in Inductive Research: Notes on the Gioia Methodology ", Organizational Research Methods, Vol. 16, $\mathrm{N}^{\circ} 1$, p. 15-31.

GOWAN M. et GATEWOOD R. (1997), «A Model of Response to the Stress of Involuntary Job Loss », Human Resource Management Review, Vol. 7, N³, p. 277-297.

GRANDEY A., DICKTER D. et HOCK-PENG S. (2004) « The customer is not always right: customer aggression and emotion regulation of service employees ", Journal of Organizational Behavior, Vol. 25, N³, p. 397-418.

IDE P. (2015). Le burn-out: une maladie du don? Nouvelle revue théologique, Vol 137, $\mathrm{N}^{\circ} 2$, p. 256-277.

JENKINS S., MITCHELL J., BAIRD S., WHITFIELD S. et MEYER H. (2011) «The Counselor's Trauma as Counseling Motivation: Vulnerability or Stress Inoculation? ", Journal of Interpersonal Violence, Vol. 26, N¹2, p. 2392-2412.

KAHN W. (1993) "Caring for the Caregivers: Patterns of Organizational Caregiving », Administrative Science Quarterly, Vol. 38, ํ․ 4, p. 539-563.

KARASEK Jr R. A. (1979). "Job demands, job decision latitude, and mental strain: Implications for job redesign". Administrative science quarterly, 285-308.

KIRK J. (1994) «Putting Outplacement in its Place », Journal of Employment Counseling, Vol. 31, p. 10-18.

LATACK J., KINICKI A. et PRUSSIA G. (1995) « An Integrative Process Model of Coping with Job Loss ", Academy of Management Review, Vol. 20, N², p. 311-342.

LEWISTON N., CONLEY J. et BLESSING-MOORE J. (1981) « Measurement of hypothetical burnout in cystic fibrosis caregivers ", Acta Paediatrica, Vol. 70, Nº6, p. 935939.

LHUILIER, D. (2010). Les «risques psychosociaux»: entre rémanence et méconnaissance. Nouvelle revue de psychosociologie, Vol. 2, p. 11-28.

LONDON M. (1998). Career Barriers: How People Experience, Overcome and Avoid Failure, Mahwah, Lawrence Erlbaum Associates.

LOUFRANI-FEDIDA S., OIRY E. et SAINT-GERMES E. (2015) «Vers un rapprochement de l'employabilité et de la gestion des compétences : grille de lecture théorique et illustrations empiriques », Revue de gestion des ressources humaines, $\mathrm{N}^{\circ}$ 97, p. 17-38.

MANGOULIA P., KOUKIA E., ALEVIZOPOULOS G., FILDISSIS G. et KATOSTARAS T. (2015) « Prevalence of Secondary Traumatic Stress Among Psychiatric Nurses in Greece », Archives of Psychiatric Nursing, Vol. 29, N5, p. 333-338.

MASLACH C. (1982). Burnout: The cost of caring. Englewood Cliffs, NJ: Prentice-Hall. 
MASLACH C. et JACKSON S. (1981) « The measurement of experienced burnout », Journal of Occupational Behavior, Vol. 2, p. 99-113.

McCANN I. et PEARLMAN L. (1990) «Vicarious traumatization: A framework for understanding the psychological effects of working with victims ", Journal of Traumatic Stress, Vol. 3, N¹, p. 131-149.

MCKEE-RYAN F., VIRICK M., PRUSSIA G., HARVEY J. et LILLY J. (2009) « Life after the layoff: getting a job worth keeping ", Journal of Organizational Behavior, Vol. 30, p. 561-580.

MCKEE-RYAN F., SONG Z., WANBERG C. et KINICKI A. (2005) «Psychological and Physical Well-Being During Unemployment: A Meta-Analytic Study », Journal of Applied Psychology, Vol. 90, ํ1, p. 53-76.

MEADOW K. (1981) "Burnout in professionals working with deaf children », American Annals of the Deaf, Vol. 156, Nº1, p. 13-22.

MEYERS T. et CORNILLE T. (2002) « The trauma of working traumatized children », In FIGLEY C. (Ed.) Treating compassion fatigue, New York: Brunner-Routledge, p. 39-55.

MONIN P. et RÜLING C.-C. (2013) «Réflexions sur la méthodologie 'à la Gioia' », Actes de la XXIIème conférence AIMS, Clermont-Ferrand, 10-12 juin.

NG W. et ROBERTS J. (2007) "'Helping the Family': The Mediating Role of Outside Directors in Ethnic Chinese Family Firms », Human Relations, Vol. 60, №2, p. 285-314.

PEARLMAN L. et MAC IAN P. (1995) «Vicarious traumatization: An empirical study of the effects of trauma work on trauma therapists ", Professional Psychology: Research and Practice, Vol. 26, p. 558-565.

PEARLMAN L. et SAAKVITNE K. (1995) Trauma and the therapist: Countertransference and vicarious traumatization in psychotherapy with incest survivors, New York: W.W. Norton.

PETERSON U., DEMEROUTI E., BERGSTROM G., ASBERG M. et NYGREN A. (2008) "Work characteristics and sickness absence in burnout and nonburnout groups: A study of Swedish health care workers », International Journal of Stress Management, Vol. 15, p. 153172. doi:10.1037/1072-5245.15.2.153

PEZET-LANGEVIN V. (2002). «Le burnout, conséquence possible du stress au travail », in NEBOIT M., VEZINA M. (Eds), Stress au travail et santé psychique, Toulouse :Octarès, p.99-109.

PRALONG J (2011). «Les projets n'engagent que ceux qui y croient! Une étude longitudinale des projets, performances et compétences», Revue française de gestion, Vol.216, nº 7 p.15-31.

REAY T. (2014) «Publishing qualitative research », Family Business Review, Vol. 27, №2, p. 95-102.

ROQUES O., ROGER A., (2004), «La gestion de la mobilité géographique des salariés : le cas de Technicatome », Revue Française de Gestion, Vol. 150, p. 71-85.

SAAKVITNE K. et PEARLMAN L. (1996) Transforming the pain: a workbook on vicarious traumatization, London: W.W. Norton.

SABIN-FARRELL R. et TURPIN G. (2003) «Vicarious traumatization: implications for the mental health of health workers? », Clinical Psychology Review, Vol. 23, p. 449-480.

SADEH N. et KARNIOL R. (2012) «The sense of self-continuity as a resource in adaptive coping with job loss », Journal of Vocational Behavior, Vol. 80, p. 93-99.

SCHAUFELI W. et GREENGLASS E. (2001) «Introduction to Special Issue On Burnout And Health », Psychology \& Health, Vol. 16, N5, p. 501-510.

SHIRI S., WEXLER I., SCHWARTZ I., KADARI M. et KREITLER S. (2010) «The association between reality-based beliefs and indirectly experienced traumatization », International Journal of Psychology, Vol. 45, Nº, p. 469-476. 
SINCLAIR H. et HAMILL C. (2007) « Does vicarious traumatisation affect oncology nurses? A literature review », European Journal of Oncology Nursing, Vol. 11, p. 348-356.

SOPER B. et VON BERGEN C. (2001) «Employment counseling and life stressors: coping through expressive writing », Journal of Employment Counseling, Vol. 38, p. 150-160.

SPERA S., BUHRFEIND E. et PENNEBAKER J. (1994) «Expressive Writing and Coping with Job Loss », Academy of Management Journal, Vol. 37, N³, p. 722-733.

TRIPPANY R., WHITE KRESS V. et WILCOXON S. (2004) «Preventing Vicarious Trauma: What Counselors Should Know When Working With Trauma Survivors ", Journal of Counseling \& Development, Vol. 82, p. 31-37.

TUSCHKE A., SANDERS G. et HERNANDEZ E. (2014) «Whose experience matters in the boardroom? The effects of experiential and vicarious learning on emerging market entry ", Strategic Management Journal, Vol. 35, N³, p. 398-418.

UCBASARAN D., SHEPHERD D., LOCKETT A. et LYON J. (2013) « Life After Business Failure: The Process and Consequences of Business Failure for Entrepreneurs », Journal of Management, Vol. 39, N¹, p. 163-202.

VRKLEVSKI L. et FRANKLIN J. (2008) «Vicarious Trauma: The Impact on Solicitors of Exposure to Traumatic Material », Traumatology, Vol. 14, Nº1, p. 106-118.

WAY I., VANDEUSEN K., MARTIN G., APPLEGATE B. et JANDLE D. (2004) «A Comparison of Clinicians Who Treat Survivors of Sexual Abuse and Sexual Offenders », Journal of Interpersonal Violence, Vol. 19, N¹, p. 49-71.

YIN R. (1990) Case Study Research: Design and Methods, Newbury Park, CA: Sage. 
ANNEXE $\mathrm{N}^{\circ} 1$ : DETAIL DES 23 ENTRETIENS SEMI-DIRECTIFS CENTRE

\begin{tabular}{|c|c|c|c|c|}
\hline $\mathbf{N}^{\circ}$ & Structures, dispositifs et publics accompagnés & Âge & Exp. & Durée \\
\hline 01 & $\begin{array}{l}60.000 \text { Rebonds - Association aidant des entrepreneurs post-faillite à rebondir } \\
\text { dans un projet entrepreneurial ou salarié }\end{array}$ & $\begin{array}{l}41 \\
\text { ans }\end{array}$ & 2 ans & $1 \mathrm{H} 14$ \\
\hline 02 & 60.000 Rebonds & $\begin{array}{l}41 \\
\text { ans }\end{array}$ & 4 ans & $1 \mathrm{H} 20$ \\
\hline 03 & $\begin{array}{l}\text { Afec - Organisme partenaire du Plie (cf. infra), spécialisé notamment dans l'aide } \\
\text { à l'orientation professionnelle et l'accompagnement à la recherche d'emploi }\end{array}$ & $\begin{array}{l}32 \\
\text { ans }\end{array}$ & 2 ans & $1 \mathrm{H} 17$ \\
\hline 04 & $\begin{array}{l}\text { Aquitaine Active - Association facilitant et sécurisant l'accès au crédit bancaire à } \\
\text { des entrepreneurs en situation d'exclusion }\end{array}$ & $\begin{array}{l}33 \\
\text { ans }\end{array}$ & 4 ans & $1 \mathrm{H} 04$ \\
\hline 05 & Avarap - Association aidant des cadres à retrouver un emploi ou à se réorienter & $\begin{array}{l}61 \\
\text { ans }\end{array}$ & 5 ans & $1 \mathrm{H} 16$ \\
\hline 06 & $\begin{array}{l}\text { Cadres Entraide - Association accompagnant et suivant des cadres en } \\
\text { reconversion ou en mutation professionnelle }\end{array}$ & $\begin{array}{l}65 \\
\text { ans }\end{array}$ & 5 ans & $1 \mathrm{H} 13$ \\
\hline 07 & Cadres Entraide & $\begin{array}{l}69 \\
\text { ans }\end{array}$ & 5 ans & $1 \mathrm{H} 32$ \\
\hline 08 & $\begin{array}{l}\text { Cap Emploi - Organisme accompagnant des personnes handicapées dans leur } \\
\text { démarche d'insertion professionnelle }\end{array}$ & $\begin{array}{l}41 \\
\text { ans }\end{array}$ & $\begin{array}{l}11 \\
\text { ans }\end{array}$ & $1 \mathrm{H} 04$ \\
\hline 09 & Cap Emploi & $\begin{array}{l}43 \\
\text { ans }\end{array}$ & $\begin{array}{l}15 \\
\text { ans }\end{array}$ & $1 \mathrm{H} 17$ \\
\hline 10 & $\begin{array}{l}\text { Ingeus - Entreprise spécialisée notamment dans la transition professionnelle des } \\
\text { salariés et l'accompagnement vers l'emploi des demandeurs d'emploi }\end{array}$ & $\begin{array}{l}37 \\
\text { ans }\end{array}$ & $\begin{array}{l}14 \\
\text { ans }\end{array}$ & $1 \mathrm{H} 02$ \\
\hline 11 & Ingeus & $\begin{array}{l}40 \\
\text { ans }\end{array}$ & 4 ans & $1 \mathrm{H} 23$ \\
\hline 12 & Jobijoba - Entreprise proposant notamment du conseil en emploi & $\begin{array}{l}34 \\
\text { ans }\end{array}$ & 9 ans & $1 \mathrm{H} 40$ \\
\hline 13 & $\begin{array}{l}\text { Parcours formations - Organisme partenaire du Plie (cf. infra) proposant } \\
\text { notamment des dispositifs d'orientation et d'insertion }\end{array}$ & $\begin{array}{l}33 \\
\text { ans }\end{array}$ & 1 an & $1 \mathrm{H} 31$ \\
\hline 14 & $\begin{array}{l}\text { Plie/Maison de l'Emploi - Dispositif favorisant l'accès ou le retour à l'emploi des } \\
\text { personnes les plus en difficulté }\end{array}$ & $\begin{array}{l}31 \\
\text { ans }\end{array}$ & 7 ans & $2 \mathrm{H} 01$ \\
\hline 15 & Plie/Maison de l’Emploi & $\begin{array}{l}35 \\
\text { ans }\end{array}$ & 9 ans & $1 \mathrm{H} 27$ \\
\hline 16 & $\begin{array}{l}\text { Pôle Emploi/Accompagnement renforcé - Dispositif de l'opérateur du service } \\
\text { public de l'emploi destiné à des personnes les plus éloignées de l'emploi }\end{array}$ & $\begin{array}{l}56 \\
\text { ans }\end{array}$ & $\begin{array}{l}13 \\
\text { ans }\end{array}$ & $1 \mathrm{H} 44$ \\
\hline 17 & Pôle Emploi/Accompagnement renforcé & $\begin{array}{l}49 \\
\text { ans }\end{array}$ & $\begin{array}{l}13 \\
\text { ans }\end{array}$ & $1 \mathrm{H} 52$ \\
\hline 18 & $\begin{array}{l}\text { Pôle Emploi/Contrat de Sécurisation Professionnelle - Dispositif de l'opérateur du } \\
\text { service public de l'emploi destiné à des salariés concernés par une procédure de } \\
\text { licenciement pour motif économique }\end{array}$ & $\begin{array}{l}53 \\
\text { ans }\end{array}$ & $\begin{array}{l}15 \\
\text { ans }\end{array}$ & 0H57 \\
\hline 19 & Pôle Emploi/Contrat de Sécurisation Professionnelle & $\begin{array}{l}62 \\
\text { ans }\end{array}$ & $\begin{array}{l}8 \\
\text { ans }\end{array}$ & $1 \mathrm{H} 42$ \\
\hline 20 & $\begin{array}{l}\text { Right Management - Entreprise accompagnant les trajectoires professionnelles } \\
\text { des individus au sein des organisations (ex. : outplacement) }\end{array}$ & $\begin{array}{l}31 \\
\text { ans }\end{array}$ & 8 ans & $1 \mathrm{H} 19$ \\
\hline 21 & Right Management & $\begin{array}{l}46 \\
\text { ans }\end{array}$ & 4 ans & $1 \mathrm{H} 13$ \\
\hline 22 & $\begin{array}{l}\text { Second Souffle - Association aidant des chefs d'entreprises de TPE/PME ayant } \\
\text { subi un échec entrepreneurial à rebondir sur une nouvelle activité ou un emploi } \\
\text { salarié }\end{array}$ & $\begin{array}{l}47 \\
\text { ans }\end{array}$ & 2 ans & $1 \mathrm{H} 30$ \\
\hline 23 & Second Souffle & $\begin{array}{l}46 \\
\text { ans }\end{array}$ & $\begin{array}{l}1,5 \\
\text { an }\end{array}$ & $1 \mathrm{H} 10$ \\
\hline
\end{tabular}


ANNEXE N'2 : CONSTRUCTION DE LA TYPOLOGIE - DETAIL DES CALCULS

\begin{tabular}{|c|c|c|c|c|c|c|c|c|c|c|}
\hline Répondant & $\begin{array}{c}\text { Cat. fig. } \\
\mathrm{N}^{\circ} 2\end{array}$ & Posture & $\begin{array}{l}\text { Périmètre } \\
\text { pro }\end{array}$ & $\begin{array}{l}\text { Périmètre } \\
\text { relationnel }\end{array}$ & $\begin{array}{l}\text { Périmètre } \\
\text { affectif }\end{array}$ & $\begin{array}{c}\text { Barrières de } \\
\text { protection }\end{array}$ & $\begin{array}{r}\text { Flexibilité } \\
\text { dispositif }\end{array}$ & $\begin{array}{c}\text { Ressources } \\
\text { externes }\end{array}$ & $\begin{array}{c}\text { Ressources } \\
\text { internes }\end{array}$ & $\begin{array}{c}\text { Effet } \\
\text { collatéral }\end{array}$ \\
\hline $\mathrm{A}$ & 1 & 3,67 & 3,00 & 5,00 & 3,00 & 3,33 & 2,00 & 4,00 & 4,00 & 2,00 \\
\hline $\mathrm{B}$ & 1 & 3,00 & 3,00 & 4,00 & 2,00 & 3,00 & 2,00 & 3,00 & 4,00 & 2,00 \\
\hline $\mathrm{C}$ & 1 & 3,33 & 2,00 & 4,00 & 4,00 & 3,33 & 2,00 & 4,00 & 4,00 & 2,00 \\
\hline $\mathrm{D}$ & 1 & 3,33 & 3,00 & 4,00 & 3,00 & 4,33 & 4,00 & 4,00 & 5,00 & 2,00 \\
\hline$E$ & 1 & 3,33 & 4,00 & 4,00 & 2,00 & 3,33 & 2,00 & 4,00 & 4,00 & 2,00 \\
\hline $\mathrm{F}$ & 1 & 4,33 & 5,00 & 4,00 & 4,00 & 4,00 & 4,00 & 4,00 & 4,00 & 2,00 \\
\hline $\mathrm{G}$ & 1 & 4,00 & 4,00 & 3,00 & 5,00 & 4,00 & 4,00 & 3,00 & 5,00 & 2,00 \\
\hline $\mathrm{H}$ & 1 & 3,33 & 3,00 & 4,00 & 3,00 & 3,67 & 3,00 & 5,00 & 3,00 & 2,00 \\
\hline I & 1 & 4,00 & 4,00 & 4,00 & 4,00 & 3,33 & 2,00 & 4,00 & 4,00 & 2,00 \\
\hline $\mathrm{J}$ & 1 & 3,00 & 4,00 & 1,00 & 4,00 & 3,33 & 4,00 & 2,00 & 4,00 & 2,00 \\
\hline $\mathrm{K}$ & 1 & 3,33 & 4,00 & 2,00 & 4,00 & 3,33 & 4,00 & 2,00 & 4,00 & 2,00 \\
\hline $\mathrm{L}$ & 1 & 3,67 & 4,00 & 4,00 & 3,00 & 4,33 & 4,00 & 4,00 & 5,00 & 2,00 \\
\hline $\mathrm{M}$ & 1 & 4,67 & 5,00 & 4,00 & 5,00 & 4,00 & 4,00 & 3,00 & 5,00 & 1,00 \\
\hline $\mathrm{N}$ & 2 & 3,00 & 3,00 & 3,00 & 3,00 & 2,33 & 1,00 & 3,00 & 3,00 & 3,00 \\
\hline $\mathrm{O}$ & 2 & 3,33 & 4,00 & 2,00 & 4,00 & 2,00 & 2,00 & 2,00 & 2,00 & 3,00 \\
\hline $\mathrm{P}$ & 2 & 4,00 & 4,00 & 5,00 & 3,00 & 2,00 & 2,00 & 2,00 & 2,00 & 3,00 \\
\hline $\mathrm{Q}$ & 3 & 2,00 & 2,00 & 3,00 & 1,00 & 2,33 & 1,00 & 3,00 & 3,00 & 4,00 \\
\hline $\mathrm{R}$ & 3 & 1,67 & 2,00 & 1,00 & 2,00 & 2,67 & 3,00 & 3,00 & 2,00 & 4,00 \\
\hline $\bar{S}$ & 3 & 1,67 & 2,00 & 1,00 & 2,00 & 2,67 & 1,00 & 3,00 & 4,00 & 3,00 \\
\hline $\mathrm{T}$ & 3 & 2,67 & 2,00 & 4,00 & 2,00 & 2,67 & 1,00 & 4,00 & 3,00 & 4,00 \\
\hline $\mathrm{U}$ & 3 & 2,67 & 2,00 & 4,00 & 2,00 & 2,33 & 1,00 & 3,00 & 3,00 & 3,00 \\
\hline $\mathrm{V}$ & 4 & 2,33 & 3,00 & 3,00 & 1,00 & 4,00 & 4,00 & 5,00 & 3,00 & 3,00 \\
\hline $\mathrm{W}$ & 4 & 1,67 & 2,00 & 2,00 & 1,00 & 3,67 & 4,00 & 3,00 & 4,00 & 4,00 \\
\hline
\end{tabular}

